\title{
UNIVERSAL INEQUALITIES FOR EIGENVALUES OF QUADRATIC POLYNOMIAL OPERATOR OF THE KOHN LAPLACIAN
}

\author{
He-Jun Sun And Xue-Rong QI
}

\begin{abstract}
In this paper, we investigate the Dirichlet weighted eigenvalue problem of quadratic polynomial operator of the Kohn Laplacian on a bounded domain in the Heisenberg group $\mathbb{H}^{n}$. We establish two inequalities for eigenvalues of this problem. One of them implies an explicit estimate for the upper bound of the $(k+1)$-th eigenvalue in terms of the first $k$ eigenvalues. Moreover, as a special case, we give some universal inequalities and estimates for eigenvalues of the bi-Kohn Laplacian.
\end{abstract}

Mathematics subject classification (2010): Primary 35P15, 53C17; Secondary 58C40.

Keywords and phrases: Eigenvalue, universal inequality, Heisenberg group, Kohn Laplacian, quadratic polynomial operator.

\section{REFERENCES}

[1] M. S. Ashbaugh And R. D. Benguria, More bounds on eigenvalue ratios for Dirichlet Laplacians in $n$ dimension, SIAM J. Math Anal. 24 (1993), 1622-1651.

[2] Q.-M. Cheng, T. ICHIKAWA AND S. MAmetsuka, Estimates for eigenvalues of a clamped plate problem on Riemannian manifolds, J. Math. Soc. Japan. 62 (2010), 673-686.

[3] Q.-M. Cheng And H. C. YAng, Estimates on Eigenvalues of Laplacian, Math. Ann. 331 (2005), 445-460.

[4] G. B. Folland And E. M. Stein, Estimates for the $\bar{\partial}_{b}$ complex and analysis on the Heisenberg group, Comm. Pure Appl. Math. 27 (1974), 429-522.

[5] K. O. Friedrichs, Spectral theory of operators in Hilbert space, Springer Verlag, New York, 1980.

[6] K. Furutani, K. SAGami And N. Ostuki, The spectrum of the Laplacian on a certain nilpotent Lie group, Comm. P. D. E. 18 (1993), 533-555.

[7] D. H. Guo, Eigenvalue problem for a class of left invariant operators on Heisenberg group, J. Beijing Norm. Univ. 28 (1992), 287-292 (in Chinese).

[8] D. H. GuO, Estimates for eigenvalues of a class of operators with variable coefficients, J. Beijing Univ. Aeron. Astron. 23 (1997), 247-251 (in Chinese).

[9] E. M. HARRELl II AND J. StubBe, On trace identities and universal eigenvalue estimates for some partial differential operators, Trans. Amer. Math. Soc. 349 (1997), 1797-1809.

[10] G. N. Hile AND M. H. Protter, Inequalities for eigenvalues of the Laplacian, Indiana Univ. Math. J. 29 (1980), 523-538.

[11] S. M. HooK, Domain independent upper bounds for eigenvalues of elliptic operator, Trans. Amer. Math. Soc. 318 (1990), 615-642.

[12] D. S. JeRISON, The Dirichlet problem for the Kohn Laplacian on the Heisenberg group I, J. Funct. Anal. 43 (1981), 97-142.

[13] J. J. KoHn, Boundaries of complex manifolds, in: Proc. Conf. Complex Analysis (Minneapolis, 1964), Springer Verlag, Berlin, 1965, 81-94.

[14] H. A. LEVINE AND M. H. PROTTER, Unrestricted lower bounds for eigenvalues for classes of elliptic equations and systems of equations with applications to problems in elastisity, Math. Mech. Appl. Sci. 7 (1985), 210-222.

[15] P. Li And S. T. YAU, On the Schrödinger equation and the eigenvalue problem, Comm. Math. Phys. 88 (1983), 309-318. 
[16] P. C. NiU And H. Q. Zhang, Payne-Pólya-Weinberger type inequalities for eigenvalues of nonelliptic operators, Pacific J. Math. 208 (2003), 325-345.

[17] G. PóLYA, On the eigenvalues of vibrating membranes, Proc. London Math. Soc. 11 (1961), 419-433.

[18] A. El Soufi, E. M. HARRELL II AND S. ILIAS, Universal inequalities for the eigenvalues of Laplace and Schrödinger operators on submanifolds, Trans. Amer. Math. Soc. 361 (2009), 2337-2350.

[19] E. M. S tein, Harmonic analysis, Princeton Univ. Press, Princeton, 1993.

[20] H. J. Sun, Yang-type inequalities for weighted eigenvalues of a second order uniformly elliptic operator with a nonnegative potential, Proc. Amer. Math. Soc. 138 (2010), 2827-2838.

[21] H. J. Sun, Q.-M. ChEng AND H. C. YANG, Lower order eigenvalues of Dirichlet Laplacian, Manuscripta Math. 125 (2008), 139-156.

[22] Q. L. WANG AND C. Y. XIA, Universal bounds for eigenvalues of the biharmonic operator on Riemannian manifolds, J. Funct. Anal. 245 (2007), 334-352.

[23] H. C. YANG, An estimate of the difference between consecutive eigenvalues, Preprint IC/91/60, 1991, Trieste, Italy. 